\title{
Juxtacrine regulation of cellular senescence
}

\author{
Masashi Narita* \\ Cancer Research UK Cambridge Institute, University of Cambridge, Cambridge CB2 ORE, UK
}

Cellular senescence is defined as a state of stable cell cycle exit in response to various stimuli, which include both cytotoxic stress and physiological cues. In addition to the core non-proliferative aspect, senescence is associated with diverse functionalities, which contribute to the role of senescence in a wide range of pathological and physiological processes. Such functionality is often mediated by the capability of senescent cells to communicate with their surroundings. Emerging evidence suggests that senescence is not a single entity, but a dynamic and heterogeneous collective phenotype. Understanding the diverse nature of senescence should provide insights into the complexity of tissue homeostasis and its disruption, such as in aging and tumorigenesis. [BMB Reports: Perspective 2019; 52(1): 3-4]

The essential feature of the autonomous aspect of senescence is stable proliferative arrest. This factor contributes to the tumor suppressive and pro-aging aspects of senescence, the latter in part mediated through limiting the replicative capacity of tissue stem cell compartments (Sharpless \& DePinho (2007) Nat Rev Mol Cell Biol 8, 703-713).

However, senescence is not simply a static endpoint. In fact, senescent cells actively communicate with their neighboring cells within the tissue microenvironment (Pérez-Mancera PA et al. (2014) Nat Rev Cancer 14, 547-558). The best example of such non-autonomous activities of senescence is the sene-

*Corresponding author. E-mail: Masashi.Narita@cruk.cam.ac.uk

https://doi.org/10.5483/BMBRep.2019.52.1.289

Received 4 November 2018

Keywords: Epigenetics, NOTCH, SAHF, SASP, Senescence

Abbreviations: ATAC-seq, Assay for Transposase-Accessible Chromatin using sequencing; ICD, intracellular domain; NIS, NOTCHinduced senescence; OIS, oncogene-induced senescence; RIS, RASinduced senescence; SAHFs, senescence-associated heterochromatin foci; SASP, senescence-associated secretory phenotype

Perspective to: Hoare M et al., 2016, NOTCH1 mediates a switch between two distinct secretomes during senescence, Nature Cell Biology 18, 979-992; Parry AJ et al., 2018, NOTCH-mediated non-cell autonomous regulation of chromatin structure during senescence, Nature Communications 9, 1840. scence-associated secretory phenotype (SASP), but other forms of cell communication have also been reported, including cell fusion, cytoplasmic bridges, and small extracellular vesicles (Chuprin A et al. (2013) Genes Dev 27, 2356-2366; Biran A et al. (2015) Genes Dev 29, 791-802; Takasugi M et al. (2017) Nat Commun 8, 15729). In addition, we have shown that $\mathrm{NOTCH}$-mediated direct cell-cell contact modulates senescence development and functionality (Diagram 1) (Hoare $\mathrm{M}$ et al. (2016) Nat Cell Biol 18, 979-992; Parry AJ et al. (2018) Nat Commun 9, 1840).

$\mathrm{NOTCH}$ proteins are conserved cell surface receptors, and $\mathrm{NOTCH}$ signaling has been implicated in diverse cellular processes, including embryonic development, cell fate and differentiation, as well as in tumorigenesis (Hoare M \& Narita M (2018) Adv Exp Med Biol 1066, 299-318). In addition, emerging evidence indicates that $\mathrm{NOTCH}$ signaling is an important senescence effector (Ito $Y$ et al. (2017) Trends Cell Biol 27, 820-832). Although enforced expression of a constitutively active form of NOTCH (the intracellular domain, ICD) can induce senescence (NOTCH-induced senescence, $\mathrm{NIS}$ ), we have shown that endogenous $\mathrm{NOTCH}$ activation during premature senescence, such as in oncogene-induced senescence $(\mathrm{OIS})$ and DNA damage-induced senescence, is transient. An early high-NOTCH phase correlates with a TGF- $\beta$-rich fibrogenic SASP, whereas a later onset low-NOTCH phase (fully established senescence) corresponds to the classical inflammatory SASP, which includes IL1, IL6 and IL8. Indeed, NOTCH signaling both activates TGF- $\beta$ and inhibits the expression of inflammatory cytokines. Therefore, the transient nature of NOTCH activity during the establishment of senescence is critical for the timely switch between the functionally distinct types of the SASP. Many collagens are typically downregulated in established senescent fibroblasts, which instead express, together with inflammatory cytokines, a number of extracellular matrix modifying enzymes. The shift from a fibrogenic to a fibrolytic SASP appears to be critical for tissue homeostasis (Demaria M et al. (2014) Dev Cell 31, 722-733; Krizhanovsky V et al. (2008) Cell 134, 657-667). It is conceivable that the persistence of the initial phase of the SASP would promote tissue fibrosis, whereas persistence of the late phase SASP would lead to chronic inflammation and delayed wound healing, if the senescent cells were not properly eliminated by the immune system. Although it remains to be tested whether $\mathrm{NOTCH}$ signaling is generally involved in the timely control of the tissue repair process 


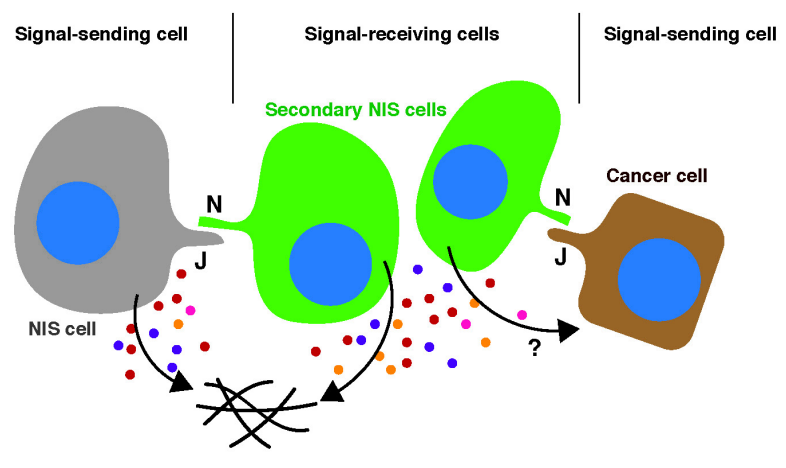

Diagram 1. Juxtacrine-mediated senescence induction. $\mathrm{NOTCH}$ ligands, such as JAG1 (J), associate with $\mathrm{NOTCH}$ receptors $(\mathrm{N})$, which then undergo a series of proteolytic cleavage events. The resulting active form of $\mathrm{NOTCH}$, the intracellular domain (ICD), is translocated to the nucleus and induces $\mathrm{NOTCH}$ responsive genes. NIS cells (signal-sending cells) can upregulate JAG1 and transfer $\mathrm{NOTCH}$ signaling to adjacent normal cells (signal-receiving cells), thereby transferring the NIS phenotype to those signal-receiving cells (secondary NIS cells) through lateral induction. JAG1-expressing cancer cells can also be signal-sending cells in this context. NIS cells secrete distinct factors, which modulate the tissue microenvironment and may also impact signal- sending cancer cells.

through senescence regulation, persistent $\mathrm{NOTCH}$ signaling has been correlated with renal fibrosis in a tissue damage model in mice (Sörensen-Zender I et al. (2014) Am J Physiol Renal Physiol 306, F907-915; Bielesz B et al. (2010) J Clin Invest 120, 4040-4054).

$\mathrm{NOTCH}$ signaling is also important for the spatial regulation of senescence. NOTCH signaling in mammals consists of five ligands and four receptors, all of which are cell surface proteins. Thus, NOTCH signaling is transmitted from signalsending cells to signal-receiving cells through direct cell contact. This is an important aspect of its role in tissue patterning. In the developmental context, two modes of signal transmission have been proposed: lateral inhibition and lateral induction (Ito $Y$ et al. (2017) Trends Cell Biol 27, 820-832). We have shown evidence that NIS signaling can be transmitted through lateral induction (Hoare $M$ et al. (2016) Nat Cell Biol 18, 979-992; Parry AJ et al. (2018) Nat Commun
9, 1840). In human fibroblast models, the NOTCH ligand JAG1 is selectively upregulated in NIS cells, which can in turn activate $\mathrm{NOTCH}$ signaling in neighboring normal cells, thereby inducing secondary NIS cells (Diagram 1). Interestingly, NIS cells are also characterized by a unique chromatin architecture. While classical oncogenic RAS-induced senescence (RIS) is often accompanied by senescence-associated heterochromatic foci (SAHFs), NIS cells lack SAHFs (Parry AJ et al. (2018) Nat Commun 9, 1840). Interestingly, at the nucleosome level, both RIS and NIS cells exhibit a substantial increase in chromatin accessibility (probed by ATAC-seq), but at different regions. In addition, when both RAS and ICD are expressed in fibroblasts, ICD appears to be dominant at least for the SASP and chromatin structure. This suggests that, senescence develops with timely and progressive alterations, not only in terms of the SASP, but also in terms of the chromatin landscape. The functional relationship between the SASP and SAHFs is not entirely clear (Aird KM et al. (2016) J Cell Biol 215, 325-334).

Since $\mathrm{NOTCH}$ ligands are often upregulated in cancer, the implication of this NOTCH-mediated juxtacrine regulation of both the SASP and chromatin structure may not be limited to senescence. Indeed, cancer cell lines expressing a high level of endogenous JAG1 (a NOTCH ligand) can induce NIS in co-cultured fibroblasts (Diagram 1) (Parry AJ et al. (2018) Nat Commun 9, 1840). It will be important to decipher how $\mathrm{NOTCH}$ orchestrates this high-level cell communication within the tissue microenvironment, between senescent and normal cells, different types of senescent cells, and between tumor and stromal cells.

\section{ACKNOWLEDGEMENTS}

This work was supported by the University of Cambridge, Cancer Research UK and Hutchison Whampoa. I thank Andrew Young for comments on this review. M.N. is supported by a Cancer Research UK Cambridge Institute core grant (C14303/A17197), a Cancer Research UK Early Detection Pump Priming award (C20/A20976), an MRC grant (MR/R010013/1), and Tokyo Tech World Research Hub Initiative (WRHI). 